\title{
CONDITIONS FOR CONTINUITY OF CERTAIN OPEN MONOTONE FUNCTIONS
}

\begin{abstract}
MELVIN R. HAGAN
Abstract. In this paper continuity of certain open monotone functions is obtained by assuming for the domain and/or range various combinations of the properties of a metric continuum, regular metric continuum, semilocal connectedness, and hereditary local connectedness. An open monotone connected function from a hereditarily locally connected separable metric continuum onto a separable metric continuum is continuous. If the domain is a regular separable metric continuum, an upper semicontinuous decomposition and resulting monotone-light factorization yield continuity of an open monotone function with closed point inverses.
\end{abstract}

By a continuum is meant a compact connected space. A function $f$ is monotone if point inverses are connected. If $f$ is a function from $X$ onto $Y$, the component decomposition $X^{\prime}$ of $X$ induced by $f$ is the collection of all components of sets of the form $f^{-1}(y)$, where $y$ varies over $Y$. A function is connected if it takes connected sets onto connected sets. A continuum is regular provided every point has arbitrarily small open neighborhoods with finite boundaries [4]. It should be noted that this is not the same as a regular topological space as usually defined.

THEOREM 1. If $f$ is an open monotone connected function from the 1 st countable space $X$ onto the 1 st countable semilocally connected space $Y$, then $f$ is continuous.

Proof. If $f$ is not continuous there exists an open set $U$ in $Y$ such that $f^{-1}(U)$ is not open in $X$. Hence there is a point $x \in f^{-1}(U)$ and a sequence $\left\{x_{n}\right\}$ of distinct points in $X-f^{-1}(U)$ such that $x_{n} \rightarrow x$. Since $Y$ is semilocally connected there exists an open set $V \subset U$ such that $Y-V$ has only a finite number of components. Since $f\left(x_{n}\right) \notin V$ for all $n$ it follows that some component $C$ of $Y-V$ contains $f\left(x_{n}\right)$ for infinitely many $n$. By Theorem 2 of $[1], f^{-1}(C)$ is connected, and $x$ is a limit point of $f^{-1}(C)$. Hence $f^{-1}(C) \cup\{x\}$ is connected but

Received by the editors October 19, 1970.

A MS 1969 subject classifications. Primary 5460; Secondary 5455.

Key words and phrases. Open monotone function, connected function, connectivity function, peripherally continuous function, regular continuum, semilocally connected, hereditarily locally connected, monotone-light factorization, upper semicontinuous decomposition. 
$f\left(f^{-1}(C) \cup\{x\}\right)=C \cup\{f(x)\}$ is not connected since $C$ is closed and $f(x) \notin C$. This contradicts $f$ being connected. Thus $f$ is continuous.

CoROLLARY 1. If $f$ is an open monotone connected function from the 1 st countable space $X$ onto the hereditarily locally connected separable metric continuum $Y$, then $f$ is continuous.

Proof. By Theorem 13.21, p. 20 of [4], $Y$ is semilocally connected.

THEOREM 2. If $f$ is an open monotone connected function from $X$ onto $Y$, where $X$ and $Y$ are separable metric continua and $X$ is hereditarily locally connected, then $f$ is continuous.

Proof. By Theorem 4 of [1], $Y$ is hereditarily locally connected. Hence by Corollary 1, $f$ is continuous.

THEOREM 3. Let $f$ be a function from the $T_{1}$ space $X$ onto the semilocally connected $T_{1}$ space $Y$ with the following properties:

(a) $f$ is finite-to-1 onto $Y$,

(b) the inverse image $f^{-1}(H)$ of a closed set $H$ in $Y$ has closed components, and

(c) if $C$ is a connected subset of $Y$, then every component of $f^{-1}(C)$ maps onto all of $C$.

Then $f$ is continuous.

Proof. If $f$ is not continuous at a point $p$ in $X$, then there is an open set $V$ containing $f(p)$ such that if $U$ is any open set containing $p, f(U)$ is not a subset of $V$. Since $Y$ is semilocally connected, there is an open set $W \subset V$ and containing $f(p)$ such that $Y-W$ has a finite number of components, $C_{1}, \cdots, C_{n}$. Now $Y-W$ closed implies that the $C_{i}$ are closed, and $f$ finite-to-1 implies that $f^{-1}\left(C_{i}\right)$ has a finite number of components $K_{i j}$, since each component maps onto all of $C_{\boldsymbol{i}}$. The point $p$ is a limit point of at least one component of $f^{-1}\left(C_{i}\right)$ for some $i$. For if for every $i, p$ is not a limit point of any component of $f^{-1}\left(C_{i}\right)$, then there is an open set $U_{i j}$ containing $p$ and disjoint from $K_{i j}$ for all $i$ and $j$. If $U$ denotes the intersection of all the $U_{i j}$, then $U$ is an open set containing $p$ such that $f(U) \cap(Y-W)$ $=\varnothing$. Thus $f(U) \subset W \subset V$. This contradicts the hypothesis that $f(U)$ is not contained in $V$ for any open set $U$. Thus $p$ is a limit point of some component of some $f^{-1}\left(C_{i}\right)$. But $p$ is not in $f^{-1}\left(C_{i}\right)$ contradicting the hypothesis that $f^{-1}\left(C_{i}\right)$ has closed components. Therefore $f$ is continuous.

THEOREM 4. If $X$ is a regular separable metric continuum and $G$ is a decomposition of $X$ into disjoint continua, then $G$ is upper semicontinuous. 
Proof. Let $\left\{D_{n}\right\}$ be a sequence of sets from $G$ and $L=\lim \sup D_{n}$. If $\left\{D_{n}\right\}$ is not a null sequence, then there is a positive number $\delta$ and a positive integer $N$ such that $n>N$ implies that $\operatorname{diam}\left(D_{n}\right)>\delta$. Since $X$ is compact, $L \neq \varnothing$. Let $p \in L$. Since $X$ is regular at $p$ there is an open set $R$ with diameter less than $\delta$ such that $F(R)$, the boundary of $R$, is finite. Since $\operatorname{diam}\left(D_{n}\right)>\delta$ for $n>N, D_{n}-R$ and $R-D_{n}$ are nonempty. If $F(R) \cap D_{n}=\varnothing$, then $D_{n}-R$ and $R-D_{n}$ form a separation for the connected set $D_{n}$. Thus $F(R) \cap D_{n} \neq \varnothing$ for all $n>N$. Since the $D_{n}$ are disjoint, $F(R)$ must contain infinitely many points. But $F(R)$ is finite. Hence $\left\{D_{n}\right\}$ must be a null sequence and $L$ is a singleton. Thus $L$ is contained in a single element of $G$ and hence $G$ is upper semicontinuous $[4, \mathrm{p} .122]$.

Theorem 5. If $f$ is a function from $X$ onto $Y$, where $X$ and $Y$ are separable metric continua, $X$ is regular, and components of sets $f^{-1}(y)$, $y \in Y$, are closed, then $f$ can be factored into the composite $f=f_{2} f_{1}$, where $f_{1}$ from $X$ onto $X^{\prime}$ is monotone and continuous and $f_{2}$ from $X^{\prime}$ onto $Y$ is light.

Proof. Since $X$ is compact, $X^{\prime}$ is a collection of disjoint continua filling up $X$. Thus, by Theorem $4, X^{\prime}$ is upper semicontinuous. Define $f_{1}$ from $X$ onto $X^{\prime}$ by $f_{1}(x)=C$ if and only if $x \in C$, and define $f_{2}$ from $X^{\prime}$ onto $Y$ by $f_{2}(C)=y$ if and only if $C$ is a component of $f^{-1}(y)$. That $f_{1}$ and $f_{2}$ have the desired properties follows as in the proof of Theorem 5 of $[1]$.

THEOREM 6. If $f$ is an open monotone function from $X$ onto $Y$, where $X$ and $Y$ are separable metric continua, $X$ is regular, and $f^{-1}(y)$ is closed for all $y \in Y$, then $f$ is continuous.

Proof. Let $f=f_{2} f_{1}$ be the factorization given in Theorem 5. Since $f$ is monotone, $f^{-1}(y)$ is a continuum and thus has only one component. Therefore $f_{2}$ is a one-to-one function from $X^{\prime}$ onto $Y$. The function $f_{2}$ is also open since if $A$ is an open set in $X^{\prime}$, then $A^{*}$ (the point set union of elements in $A)$ is open in $X$. Thus $f\left(A^{*}\right)=f_{2}\left(f_{1}\left(A^{*}\right)\right)$ $=f_{2}(A)$ is open in $Y$ since $f$ is an open function. Hence $f_{2}$ is an open function. Since $f_{2}$ is an open one-to-one function, $f_{2}^{-1}$ is a continuous function from $Y$ onto $X^{\prime}$. But $X^{\prime}$ is a compact metric space. Therefore $\left(f_{2}^{-1}\right)^{-1}=f_{2}$ is continuous $[4$, p. 25]. Hence $f$ is the composite of two continuous functions and thus is continuous.

CoRollary 2. If $f$ is an open monotone function from $X$ onto $Y$, where $X$ and $Y$ are separable metric continua, $X$ is regular and $f$ is either a connected, connectivity, or peripherally continuous function, then $f$ is continuous. 
Proof. For connected, connectivity, and peripherally continuous functions, point inverses have closed components [2], [3]. Thus if $f$ is monotone, $f^{-1}(y)$ is closed for each $y \in Y$.

THEOREM 7. If $f$ is an open monotone connected function from the space $X$ onto the semilocally connected 1 st countable space $Y$, and if $X^{\prime}$ is upper semicontinuous, then $f$ is continuous.

Proof. Just as in the proof of Theorem $6, f$ can be factored into the composite $f=f_{2} f_{1}$, where $f_{1}$ is monotone and continuous from $X$ onto $X^{\prime}$ and $f_{2}$ is one-to-one and open from $X^{\prime}$ onto $Y$. Just as in the proof of Theorem 5 of [1], $f_{2}$ is a connected function since $f$ is connected. By Theorem 2 of [1], $f_{2}^{-1}$ is a connected function. Therefore $f_{2}$ is a biconnected function and by Theorem 3.7 of [3] is continuous. Thus, $f$ is continuous.

The author wishes to acknowledge helpful comments made by the referee.

\section{REFERENCES}

1. M. R. Hagan, A note on connected and peripherally continuous functions. Proc. Amer. Math. Soc. 26 (1970), 219-223.

2. P. E. Long, Properties of certain non-continuous transformations, Duke Math. J. 28 (1961), 639-645. MR 24 \#A2945.

3. — Connected mappings, Duke Math. J. 35 (1968), 677-682. MR 38 \#2745.

4. G. T. Whyburn, Analytic topology, Amer. Math. Soc. Colloq. Publ., vol. 28, Amer. Math. Soc., Providence, R. I., 1963. MR 32 \#425.

North Texas State University, Denton, Texas 76201 